\title{
Outcome of Thy 3 Thyroid Nodules: An Audit
}

P Wong, $\mathrm{H}$ Kaddour

Department of Ear, Nose and Throat Surgery, Queen's Hospital, Barking, Havering, and Redbridge University NHS Trust, UK

\section{Background:}

The British Thyroid Associated guidelines for management of thyroid cancer recommends ultrasound grading classification and Thy classification for assessment of thyroid nodules.

\section{Objectives:}

The aim of this audit is to evaluate the outcome of Thy 3 thyroid nodules and to assess the multidisciplinary team adherence to BTA guidelines recommendation.

\section{Methods:}

A retrospective review of cytology, ultrasound report and histology of 73 patients with Thy 3 cytology between 2014 and 2017 was performed. 23 were excluded as did not undergo surgery for various reasons.

\section{Results:}

The rate of malignancy in Thy 3 thyroid nodules is $24 \%$ (all Thy $3 F$ ) consistent with BTA guideline. Only $34 \%$ had U-grading and only $66 \%$ had nodule size measured which is important in risk assessment in the ultrasound reports. $30 \%$ were downgraded following multidisciplinary discussion.

\section{Discussion:}

Majority of ultrasound scans with no U-grading or size measurement were performed by sonographers. Some scans needed repeated by head and neck radiologist

Cytology should be reported by pathologist with thyroid interest as $30 \%$ downgraded at MDT meeting

\section{Conclusion:}

It is important to audit thyroid multidisciplinary team's adherence to the BTA guidelines in order to ensure best quality care is provided. 